\title{
Herlyn-Werner-Wunderlich syndrome complicated with pyocolpos: an unusual cause of postabortal sepsis
}

\author{
Deepti Sharma*, M. K. Janu, Ramesh Gaikwad, M. G. Usha
}

Department of Obstetrics \& Gynecology, Amrita Institute of Medical Sciences, Kochi-682041, Kerala, India

Received: 15 December 2012

Accepted: 6 January 2013

*Correspondence:

Dr. Deepti Sharma

E-mail: sdeepti108@gmail.com

\begin{abstract}
Obstructive mullerian anomalies give rise to a spectrum of clinical presentations and are uncommon in routine gynecologic practice. The patient usually becomes symptomatic in early reproductive years. Recurrent pelvic pain, dysmenorrhoea, enlarging abdominopelvic mass and abnormal vaginal discharge are the common presenting symptoms. We describe a rare case of a mullerian anomaly getting diagnosed 13 years after attaining menarche during evaluation of postabortal sepsis. Patient presented two weeks following evacuation done for missed abortion, with acute abdominal pain, fever and foul smelling discharge per vaginum. The anomaly was identified as uterus didelphys with obstructed left hemivagina and ipsilateral renal agenesis (Herlyn-Werner-Wunderlich syndrome) complicated by pyocolpos. She was successfully managed by single stage transvaginal septum resection under laparoscopic control.
\end{abstract}

Keywords: Herlyn-Werner-Wunderlich syndrome, Pyocolpos, Diagnostic laparoscopy

\section{INTRODUCTION}

Uterus didelphys with obstructive hemivagina and ipsilateral renal anomaly is a rare congenital abnormality of the female urogenital tract. ${ }^{1}$ It is difficult to estimate the exact incidence of obstructive Mullerian anomalies but is reported to be around 0.1 to $3.8 \%{ }^{2}$ The anomaly is difficult to diagnose due to its variable clinical presentation and variations in the age at the time of diagnosis. It may go undetected in the early reproductive years and may come to light after commencement of sexual activity or when childbearing is attempted. The time of diagnosis and clinical presentation can be influenced by the level and degree of completeness of the uterovaginal obstruction.

\section{CASE REPORT}

A 26 years old, nulliparous lady presented to gynecology department with lower abdominal pain, fever and foul smelling discharge per vaginum since two days. She had undergone dilatation and evacuation for missed abortion two weeks back. It was a spontaneous conception, two years following marriage, and early pregnancy evaluation at six weeks revealed a viable singleton pregnancy in the right horn of a bicornuate uterus and a $9 \times 7 \mathrm{~cm}$ cystic lesion with internal echoes in the region of left adnexa, posterior to urinary bladder. Repeat scan at 8 weeks revealed absent cardiac activity and evacuation was done at a local hospital. Immediate postabortal period was uneventful.

She was febrile with significant tachycardia and no pallor. Abdominal examination revealed tenderness in left iliac fossa and hypogastrium with no palpable mass. Vulval inspection revealed asymmetry at the introitus with a bulge on the left side and unhealthy vaginal discharge. Swab was taken for C\&S. Per speculum examination not done due to severe tenderness. On bimanual examination cervix felt high up and to the right side, cephalad and posterior to a tender cystic mass of about $10 \times 8 \mathrm{~cm}$ on the left side, uterine body was shifted to right and normal in size. Lab parameters showed normal $\mathrm{Hb}$, TLC-12,500 with neutrophilia, and highly elevated CRP. USG was reported as uterus didelphys (as the uterine horns were widely separated) with a large collection of $10.3 \times 6.8 \mathrm{~cm}$ with pockets of air seen on the left side, posterolateral to the bladder, normal ovaries 
and absent left kidney. An obstructive mullerian anomaly was suspected and MRI was done to confirm the same (Figure 1a-c).

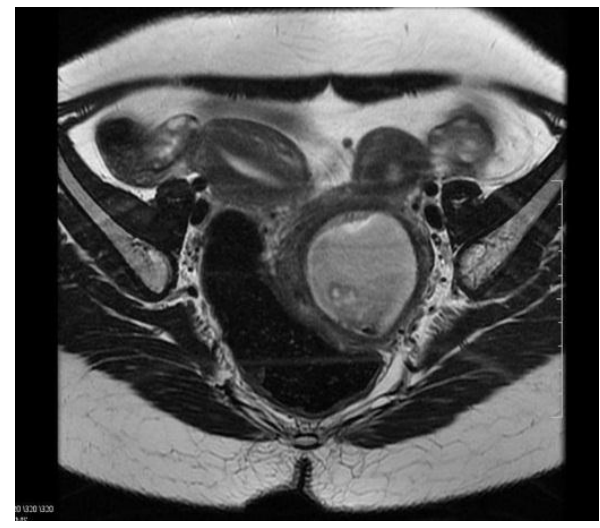

(a)

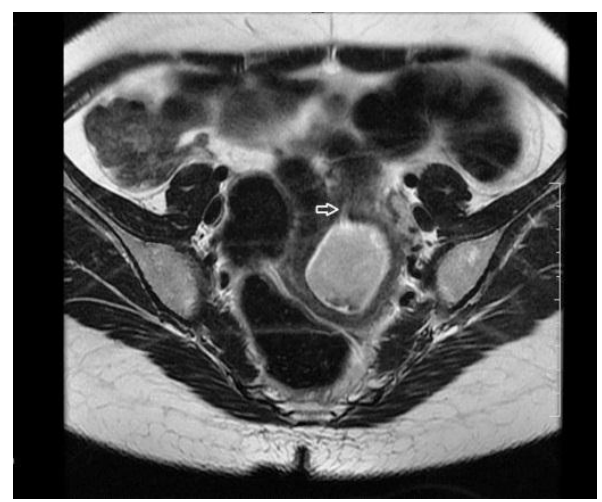

(b)

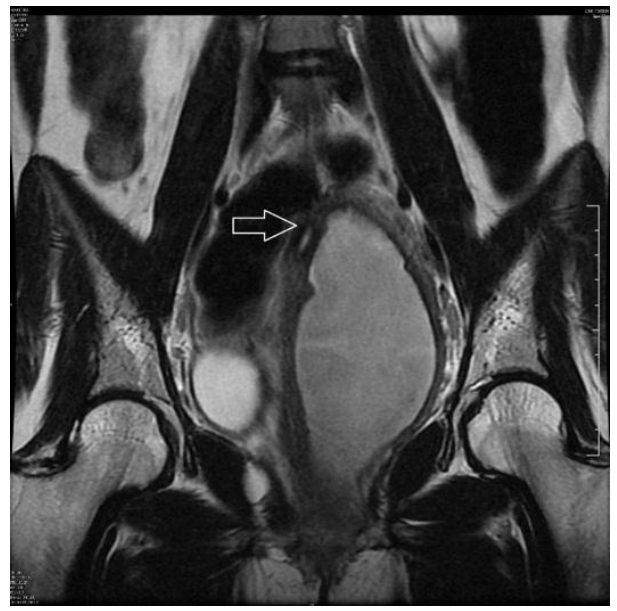

(c)

Figure 1: a. MRI pelvis (axial image) - Uterus didelphys, normal endometrial cavity, normal ovaries, with large collection in left hemivagina, b. Open arrow showing communication of collection with left uterine body and c. Coronal image - Collection compressing the normal patent vagina on right side ( open arrow).

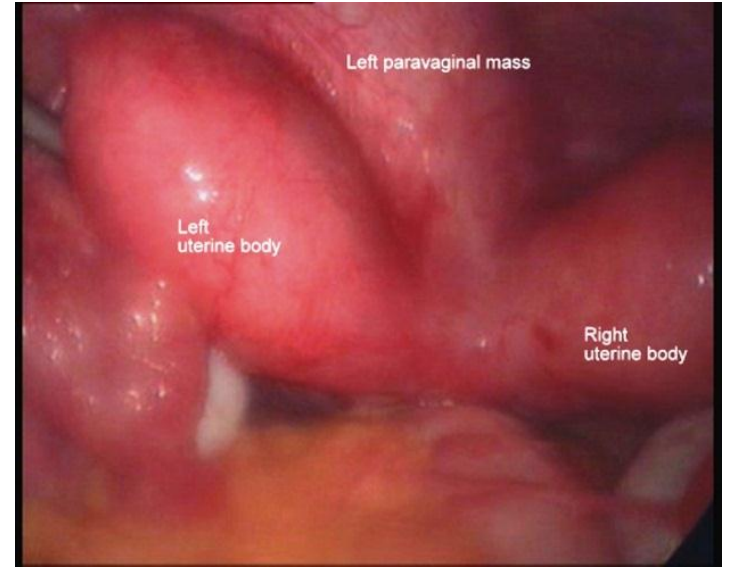

(a)

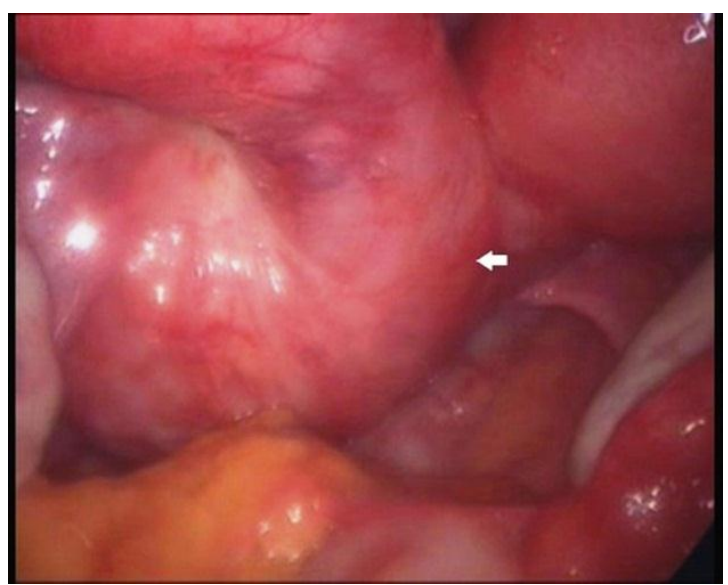

(b)

Figure 2: a. Laparoscopic view - Both uterine bodies with adnexa are normal. No pelvic collection or purulent exudate through the tubes and $b$. Posterior view of the collection.

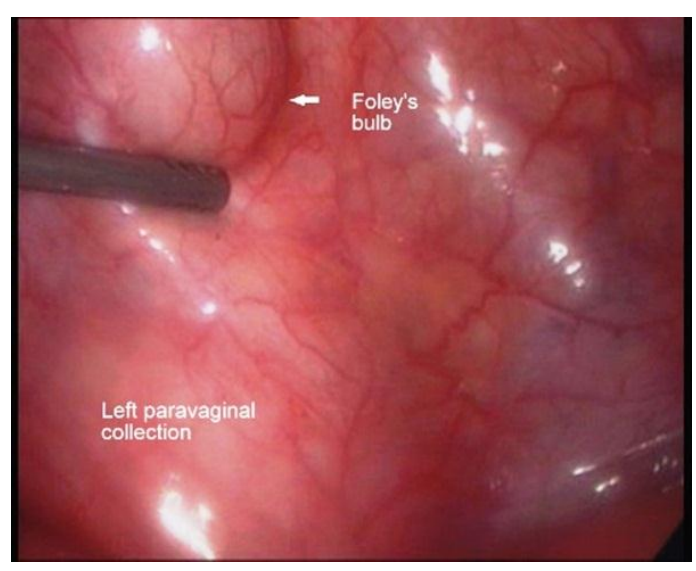

Figure 3: Anatomical limits of collection identified in relation to the bladder.

Patient was started on antibiotics and planned for surgery. Local examination findings were reconfirmed before starting the procedure. Left lateral vaginal mass was 
palpated and Foley's catheter placed to define the limits of the bladder. There was a tiny fistulous opening on the vaginal septum $4 \mathrm{~cm}$ above the hymenal ring. Diagnostic laparoscopy was done (Figure 2a-b). The extent of the left paravaginal mass noted and limits of the bladder were identified (Figure 3). Cruciate incision was made transvaginally, at the site of the tiny fistulous opening on the longitudinal vaginal septum, and around $200 \mathrm{cc}$ of foul smelling, frothy, purulent material drained. Specimen was sent for C\&S. Digital palpation through the cruciate incision helped in defining the extent of the vaginal septum. It was excised using electrocautery until the left cervix was reached. Fine absorbable sutures were placed on the resected septum to attain hemostasis. Postoperative period was uneventful and she conceived three months after surgery.

\section{DISCUSSION}

The specific association of uterus didelphys, obstructed hemivagina with ipsilateral renal anomaly is well recognized. $^{3}$ The triad has been described as HerlynWerner-Wunderlich syndrome. ${ }^{4,5}$ More recently, the acronym OHVIRA is used to describe patients with obstructed hemivagina and ipsilateral renal anomaly, that is, occurrence of two out of the three components of the triad. ${ }^{6}$ It allows inclusion of uterine anomalies other than uterus didelphys, like septate or bicornuate uterus, which are estimated to occur in about $20 \%$ of such cases.

Uterus didelphys results due to complete failure of lateral fusion of the paramesonephric ducts. Incomplete fusion and resorption leads to bicornuate or septate uterus which are common accompaniments in OHVIRA syndrome. The occurrence of partial or complete vaginal septum is due to vertical fusion defect of paramesonephric ducts with the urogenital sinus. Associated arrest in the development of the metanephric ducts leads to a variety of renal tract abnormalities. These include renal agenesis with or without agenesis of the ureter, duplication of the ureters, or multicystic dysplastic kidney. ${ }^{7}$

The obstructed hemivagina leads to development of hematometrocolpos and secondary effects of reverse menstruation like endometriosis, and pelvic adhesions subsequently. These may have a negative impact on future fertility. Recurrent cyclic abdominal pain, dysmenorrhoea, enlarging lower abdominal mass, ${ }^{3}$ coital difficulty, and abnormal vaginal discharge are various presenting symptoms. The pocketed vaginal collection with degenerated blood products can get secondarily infected giving rise to symptoms of pelvic inflammatory disease. $^{2}$ The patient often gives history of regular normal menstrual cycles and can be asymptomatic for years after menarche. ${ }^{8}$ Diagnosis can therefore be elusive and requires high degree of clinical suspicion.

Among the imaging modalities, MRI is considered as the gold standard in the diagnosis of Mullerian anomalies. ${ }^{2}$ The multiplanar imaging helps to delineate the uterovaginal anatomy with high accuracy. ${ }^{7}$ In addition, it aids in identifying associated tuboovarian pathologies, characterizing the thickness and location of the septum, the type of collection in the obstructed hemivagina, and associated malformations of the urinary tract which commonly coexist with Mullerian anomalies.

The treatment is aimed at the complete resection of the vaginal septum and establishing continuity of the obstructed side. Transvaginal resection can be performed either as a single or a double stage procedure. ${ }^{6}$ Laparoscopy prior to resection helps to rule out additional adnexal pathology and confirm the diagnosis if preoperative MRI is not feasible. Resection under laparoscopic control also helps to avoid or detect an inadvertent bladder injury as the paravesical anatomy is distorted in such cases. Hysteroscopic vaginal septum resection under transabdominal ultrasound guidance is preferable in young females in order to preserve the hymenal integrity. ${ }^{9}$ The surgical outcome is excellent and associated with successful reproductive performance in future life. ${ }^{2,4,10}$

\section{Funding: No funding sources}

Competing interests: There are no competing interests to declare

Ethical approval: Not required

\section{REFERENCES}

1. Dhar H, Razek YA, Hamdi I. Uterus Didelphys with Obstructed Right Hemivagina, Ipsilateral Renal Agenesis and Right Pyocolpos: A Case Report. Oman Med J 2011;26:447-50.

2. Burgis J. Obstructive Müllerian anomalies: Case report, diagnosis, and management. Am J Obstet Gynecol 2001;185:338-44.

3. Jindal G, Kachhawa S, Meena GL, Dhakar G. Uterus didelphys with unilateral obstructed hemivagina with hematometrocolpos and hematosalpinx with ipsilateral renal agenesis. J Hum Reprod Sci 2009;2:87-9.

4. Gholoum S, Puligandla PS, Hui T, Su W, Quiros E, Laberge JM. Management and outcome of patients with combined vaginal septum, bifid uterus, and ipsilateral renal agenesis (Herlyn-WernerWunderlich syndrome). J Pediatr Surg 2006;41:98792.

5. Orazi C, Lucchetti MC, Schingo PM, Marchetti P, Ferro F. Herlyn-Werner-Wunderlich syndrome: uterus didelphys, blind hemivagina and ipsilateral renal agenesis. Sonographic and MR findings in 11 cases. Pediatr Radiol 2007;37:657-65.

6. Smith NA, Laufer MR. Obstructed hemivagina and ipsilateral renal anomaly (OHVIRA) syndrome: management and follow-up. Fertil Steril 2007;87:918-22.

7. Han B, Herndon CN, Rosen MP, Wang ZJ, DaldrupLink H. Uterine didelphys associated with obstructed 
hemivagina and ipsilateral renal anomaly (OHVIRA) syndrome. Radiology Case Reports 2010;5:327.

8. Varras M, Akrivis Ch, Karadaglis S, Tsoukalos G, Plis Ch, Ladopoulos I. Uterus didelphys with blind hemivagina and ipsilateral renal agenesis complicated by pyocolpos and presenting as acute abdomen 11 years after menarche: presentation of a rare case with review of the literature. Clin Exp Obstet Gynecol 2008;35:156-60.
9. Kim TE, Lee GH, Choi YM, Jee BC, Ku SY, Suh $\mathrm{CS}$, et al. Hysteroscopic resection of the vaginal septum in uterus didelphys with obstructed hemivagina. J Korean Med Sci 2007;22:766-9.

10. Raga F, Bauset C, Remohi J, Bonilla-Musoles F, Simón C, Pellicer A. Reproductive impact of congenital Müllerian anomalies. Hum Reprod 1997;12:2277-81.

DOI: $10.5455 / 2320-1770 . i j r \operatorname{cog} 20130216$

Cite this article as: Sharma D, Janu MK, Gaikwad R, Usha MG. Herlyn-Werner-Wunderlich syndrome complicated with pyocolpos: an unusual cause of postabortal sepsis. Int J Reprod Contracept Obstet Gynecol 2013;2:88-91. 